\title{
Effect of Body Positions on Quadriceps Angle Measurement
}

\author{
Swati Paranjape ${ }^{a^{*}}$, Nikita Singhania ${ }^{\text {a }}$ \\ ${ }^{a}$ Physiotherapy Department, Seth G S Medical College, Parel Mumbai, India.
}

Received 07 January 2019; Accepted 21 February 2019

\begin{abstract}
Background: Quadriceps angle or Q angle is an index of the vector for the combined pull of extensor mechanisms and patellar tendon. It is a clinical measurement for knee and patella alignment. It is not a static but rather a dynamic consequence to the muscle mechanics, leg loading, and foot and leg position. Therefore in present study we assessed whether change of body position i.e. standing and supine position affects the Q angle measurement. Aims and Objectives: Present study aimed at finding whether there are any variations in $\mathrm{Q}$ angle in standing and supine position. Objectives of the study were to measure $\mathrm{Q}$ angle in supine position and standing position. We intended to assess the variations in the $\mathrm{Q}$ angle with change of body position. Methods: In this cross sectional analytical study, we assessed female participants between age group of 18-25 years, in supine and standing position, Q angle was measured in both the positions. Sample size was 100. Results: The results showed statistically significant increase in $\mathrm{Q}$ angle in standing position as compared with the supine position ( $\mathrm{p}<0.0001$ ) (C.I. 1.720 to 3.140). Conclusion: There was statistically significant increase in the Q angle measurement in standing as compared with the measurements in supine. Measuring the $\mathrm{Q}$ angle is concerned with assessing the knee functions during daily activities and sports participation, therefore assessing this important measurement not only in supine but also in a weight-bearing functional position in clinical setups would be helpful.
\end{abstract}

Keywords: Q Angle; Change in Body Position; Standing; Supine Lying; Females.

\section{Introduction}

The quadriceps angle or Q angle is the angle formed by the intersection of lines, one joining the anterior superior iliac spine (ASIS) and going to the center of the patella, and another from the tibial tuberosity to the center of patella. It is a clinical measure commonly used to assess knee alignment with respect to the hip, femur and tibia, as well as evaluating the alignment of the patella [1].

$\mathrm{Q}$ angle is an index of the vector for the combined pull of extensor mechanisms and patellar tendon [2]. It is a clinical measurement for knee and patella alignment. It is not a static but rather a dynamic consequence to the muscle mechanics, leg loading and foot and leg position which differs in both the positions [3]. Melicharek and others (2011) found that a dynamic Q-angle measurement should be taken as static measurements may not provide an accurate predictor of the force vectors between the quadriceps femoris and the patellar ligament during weight-bearing activities [3]. There are other studies which have assessed variations in Q angle with respect to gender, hip rotation etc. According to Sanchez and others (2014), there are differences in the value of the Q angle between positions with internal and external rotation of the lower limb in orthostatic position. However, when comparing the Q angle in the supine posture no statistical differences were found i.e. the values of the Q angle did not change with the limb rotation in this position, suggesting that because of the absence of muscle contraction promoting muscle relaxation and release of the patellofemoral joint [4]. It was found that there could be within subject and between gender asymmetry in Q angle. It may also vary with the change in limb position. Significant positive correlation was found between $Q$ angle

* Corresponding author: swati.paranjape@kem.edu

\section{doi http://dx.doi.org/10.28991/SciMedJ-2019-0101-3}

$>$ This is an open access article under the CC-BY license (https://creativecommons.org/licenses/by/4.0/).

(C) Authors retain all copyrights. 
and weight of individual [5]. Q angle has been identified as a potential risk factor for knee injuries, the relationship among alignment characteristics may help clinicians effectively identify those that may be at greater risk for injury and therefore help us develop intervention strategies to subsequently reduce the risk of a lower extremity injury [6].

Therefore in the present study we intended to find whether there are any variations in Q angle measurements in standing and supine position. Our outcome measures were $Q$ angle in degrees in supine position, Q angle in degrees in standing position. We assessed the variations in ' $Q$ ' angle in both positions.

\section{Methods}

In this cross sectional analytical study we recruited healthy female participants between age group of 18 to 25 years who were willing to participate in the study. Females with any musculoskeletal abnormality like fracture, dislocations, surgery, recent injury, congenital deformities or neurological conditions were excluded from the study participation. Written informed consent was taken from each participant and demographic data was recorded for each participant which included age, height, weight.

\section{Study Procedure}

Each participant was asked to stand on a raised platform with feet together in Rhombergs position [3]. All the anatomical markings for ASIS, midpoint of patella and tibial tuberosity were marked using coloured marker. Different colour markers were used for standing and supine Q angle measurements. A line was drawn using a scale from the ASIS to the midpoint of the patella and from the tibial tuberosity to the midpoint of patella. The angle formed by these two intersecting lines is the Q angle. This was measured using a universal goniometer. The participant was then asked to lie down in supine position with neutral joint alignment [4]. Again the anatomical landmarks as above were marked and Q angle was measured. Sequencing of assessing Q angle either in supine or standing was randomly allocated.

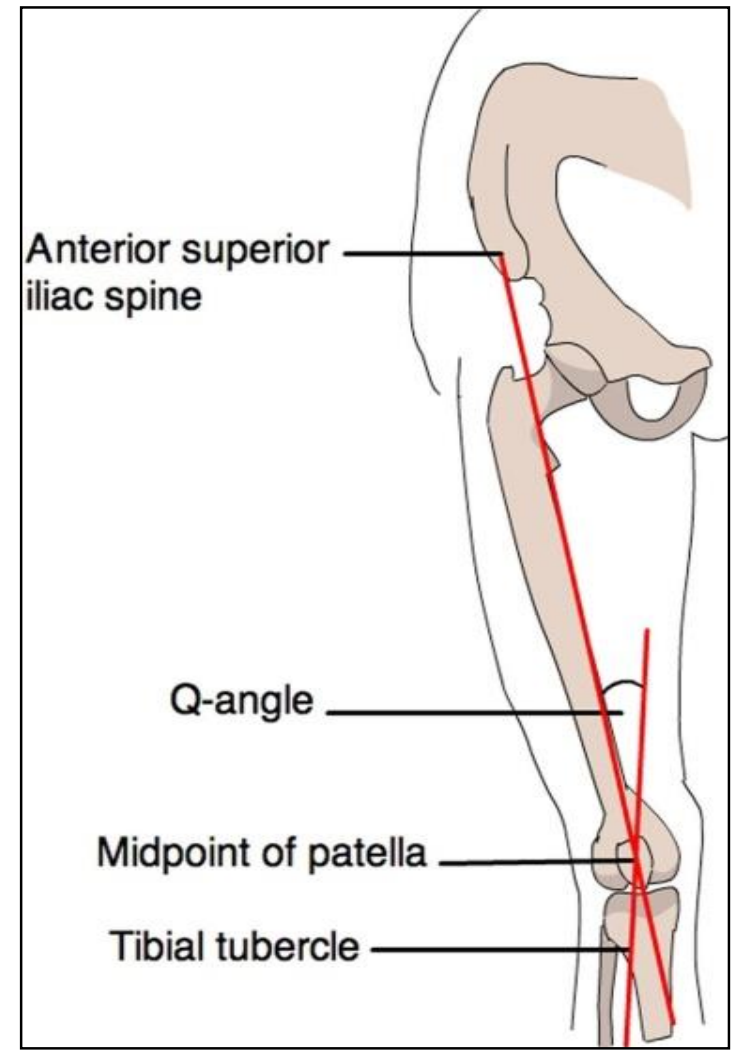

Figure 1. $Q$ angle

\section{Results}

Collected data were analysed on Graphpad Prism software. Sample was tested for normality. Paired ' $t$ ' test was used to analyse results as sample was normally distributed.

Our participants were females between the age group of 18-25 years. Mean age was 20.4 years. The mean value of supine and standing position was 20.46 and 22.87 respectively. The standard deviation for supine and standing position was 4.31 and 3.77 respectively. The confidence interval for supine position was 19.6-21.32 and for standing position was 22.13-23.65. Table 1 shows the comparison of means between two readings. Figure 2 shows the 
percentile distribution of the data for Q angle measurements in supine and standing position.

Table1. Comparison of mean and SD of $Q$ angle measurement in supine and standing position

\begin{tabular}{ccc}
\hline & Supine & Standing \\
\hline Mean & 20.46 & 22.87 \\
SD & 4.31 & 3.77 \\
CI & $19.6-21.32$ & $22.13-23.65$ \\
\hline
\end{tabular}

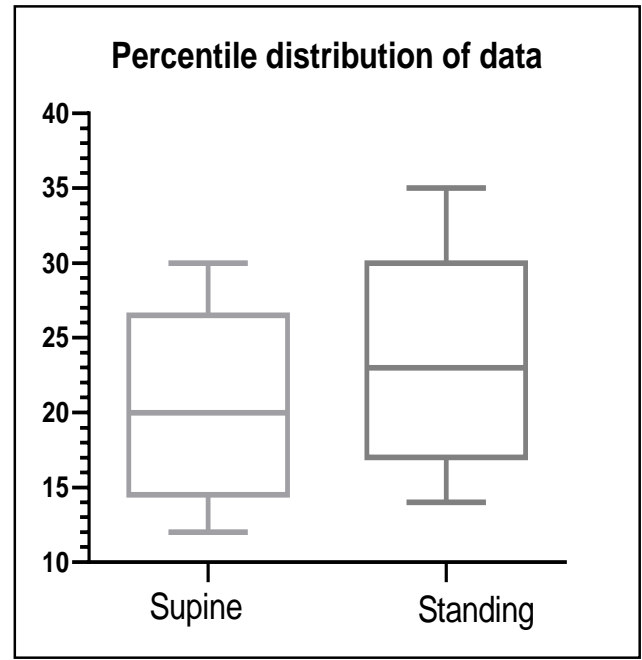

Figure 2. Percentile distribution of $Q$ angle in supine and standing position

Graphical representation of mean and standard deviation of supine and standing position is shown in Figure 3.

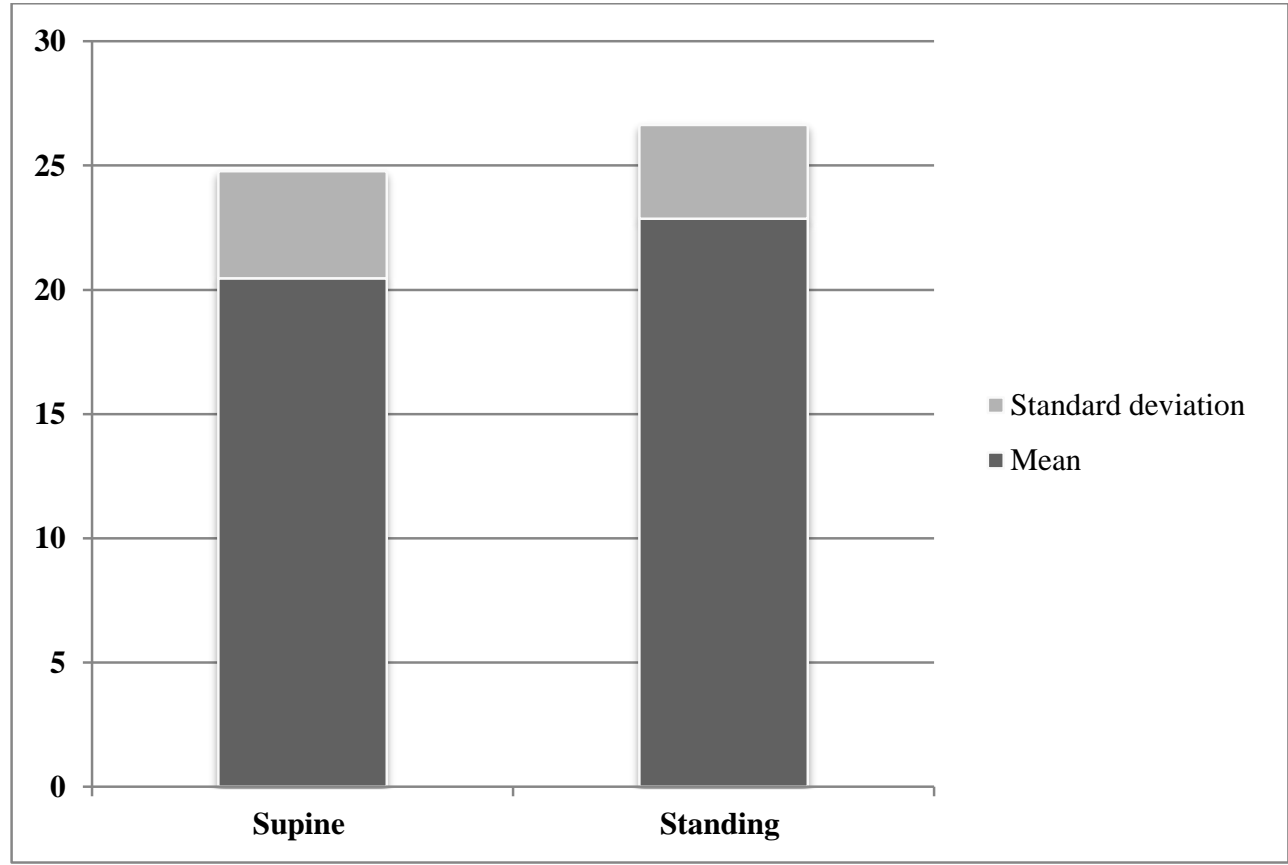

Figure 3. Mean and SD of $Q$ angle measurement in supine and standing position

The paired't' test showed highly significant increase in $\mathrm{Q}$ angle in standing position as compared with measurements in supine position. The $\mathrm{p}$ value was $<0.0001$. Difference of mean was 2.43 . Confidence interval was 1.720 to 3.140 .

\section{Discussion}

The primary finding of the study was that the $\mathrm{Q}$ angle differs with different positions of the body showing an 
increase in $\mathrm{Q}$ angle in standing position. The finding supports our hypothesis as there was statistically significant difference in $\mathrm{Q}$ angle measurement while taken in supine and standing position. Q angle was found to be increased significantly in standing position.

Q angle differs with the muscle work required in both the static postures. In supine position most of the muscles are relaxed with neutral joint alignment. The probable influencing factor could be change in the position of the patella with change in body position. The patella shows obvious movement superiorly and laterally when the quadriceps muscle contracts isometrically [7]. The significant effect of the contraction condition demonstrates the need to address the state of quadriceps contraction or relaxation in order to standardize the test procedures [7]. In standing position the lower limb antigravity muscles like gluteal muscles, quadriceps, adductors, hamstrings, gastrocnemius and soleus have to work against the gravitational force to maintain the position [8].

Increase in $\mathrm{Q}$ angle signifies effect of many factors of the lower limb kinetic chain like the pelvic angle, femoral anteversion, tibial torsion, tibiofemoral angle, foot position [2]. Valgus angle formed by the anatomical axes of the femur and tibia would move the patella medially relative to ASIS and tibial tuberosity laterally thus increasing the Q angle. Also femoral anteversion represents medial torsion of femur as femoral neck is projected forward relative to femoral condyles resulting in medial displacement of patella causing an increase in Q angle. Hip internal rotation would displace the anatomical axes of femur into adduction and tibia into abduction, increasing the tibiofemoral angle and thus increasing the $\mathrm{Q}$ angle.

Wider pelvis has potential to increase valgus at knee joint, as the angulation of femur in frontal plane would have to be greater to maintain normal stance width. Excessive pronation causes internal tibial rotation which leads to increase in knee valgus causing an increase in Q angle measurement [9].

As mentioned earlier $Q$ angle has been identified as potential risk factor and predictor of knee injury [6]. An increase in $\mathrm{Q}$ angle beyond the normal range is considered as indicative of extensor mechanism misalignment, and has been associated with patellofemoral pain syndrome, knee joint hypermobility and patellar instability [10]. Measuring $\mathrm{Q}$ angle is concerned with assessing the knee functions during daily activities and sports participation, it certainly makes sense to obtain this important measurement while in a weight-bearing position in clinical setups [5].

These results however could not be generalized in age matched male population as the study population was females. Effect of individual factors affecting Q angle could not be commented upon as their effect was not studied.

\section{Conclusion}

It can be concluded from the study that there is an increase in the Q angle measurement in standing position as compared with supine position. These findings also hold potential for future research regarding the effect of individual limb loading factors on $\mathrm{Q}$ angle measurements in standing position. From the above conclusion it may be suggested that Q angle should be measured not only in supine but also in standing position which is the functional position. It may help in better estimate of the force vector of quadriceps muscle.

\section{Acknowledgements}

We gratefully acknowledge all the participants who participated in this study.

\section{Declaration of Competing Interest}

The authors declare that they have no known competing financial interests or personal relationships that could have appeared to influence the work reported in this paper.

\section{References}

[1] Sanchez, H. M. (2014). Assessment of Patellar Alignment in Different Postures. American Journal of Sports Science, 2(2), 3034. doi:10.11648/j.ajss.20140202.14

[2] Nandi (Kar), M., Deb, S., Pal, J. N., Tapadar, A., Roy, H., \& Kar, C. (2013). The "quadriceps angle": correlation between clinical and radiographic measurements from a study in North Bengal. Journal of the Anatomical Society of India, 62(1), 68-72. doi:10.1016/s0003-2778(13)80016-0

[3] Melicharek, A., Miller, M. K., \& Hazzard, J. (2011). A Comparison of the Bilateral, Dynamic Q-Angle in Females. In ISBSConference Proceedings Archive (Vol. 1, No. 1).

[4] Sanchez, H. M., Sanchez, E. G. de M., Baraúna, M. A., \& Canto, R. S. de T. (2014). Evaluation of Q angle in differents static postures. Acta Ortopédica Brasileira, 22(6), 325-329. doi:10.1590/1413-78522014220600451.

[5] Maharjan, R., Shrestha, B., Khanal, G., Chaudhary, P., \& Karn, N. (2013). Quadriceps angle in eastern Nepalese population. Health Renaissance, 11(2), 150-156. doi:10.3126/hren.v11i2.8223. 
[6] Nguyen, A.-D., Boling, M. C., Levine, B., \& Shultz, S. J. (2009). Relationships Between Lower Extremity Alignment and the Quadriceps Angle. Clinical Journal of Sport Medicine, 19(3), 201-206. doi:10.1097/jsm.0b013e3181a38fb1.

[7] Guerra, J. P., Arnold, M. J., \& Gajdosik, R. L. (1994). Q Angle: Effects of Isometric Quadriceps Contraction and Body Position. Journal of Orthopaedic \& Sports Physical Therapy, 19(4), 200-204. doi:10.2519/jospt.1994.19.4.200.

[8] Letafatkar, A. (2013). Flat Foot Deformity, Q Angle and Knee Pain are interrelated in Wrestlers. Journal of Novel Physiotherapies, 03(02). doi:10.4172/2165-7025.1000138.

[9] Daneshmandi, H., Saki, F., Shahheidari, S., \& Khoori, A. (2011). Lower extremity Malalignment and its linear relation with Q angle in female athletes. Procedia - Social and Behavioral Sciences, 15, 3349-3354. doi:10.1016/j.sbspro.2011.04.298.

[10] Raveendranath Raveendranath, S. N., Sujatha, N., Priya, R., \& Rema, D. (2011). Bilateral variability of the quadriceps angle (Q angle) in an adult indian population. Iranian journal of basic medical sciences, 14(5), 465. doi: 10.22038/IJBMS.2011.5044. 